\title{
The atheroprotective roles of heart-protecting musk pills against atherosclerosis development in apolipoprotein E-deficient mice
}

\author{
Li Lu ${ }^{1 \#}$, Yating Qin ${ }^{1 \#}$, Chen Chen ${ }^{2}$, Xinxin Zhang ${ }^{1}$, Xiangyu Xu ${ }^{3}$, Chao Lv ${ }^{1}$, Xiaoning Wan ${ }^{1}$, Weibin Ruan ${ }^{1}$ \\ Xiaomei Guo ${ }^{1}$ \\ ${ }^{1}$ Department of Cardiology, Tongji Hospital, Tongji Medical College, Huazhong University of Science and Technology, Wuhan 430030, China; \\ ${ }^{2}$ Department of Cardiology, The Third People's Hospital of Hubei Province, Wuhan 430030, China; ${ }^{3}$ Department of Cardiology, The Second \\ Hospital of Shandong University, Jinan 250000, China \\ Contributions: (I) Conception and design: L Lu, Y Qin, X Guo; (II) Administrative support: X Guo; (III) Provision of study materials or patients: \\ C Chen, X Xu; (IV) Collection and assembly data: X Zhang, C Lv, X Wan, W Ruan; (V) Data analysis and interpretation: All authors; (VI) \\ Manuscript writing: All authors; (VII) Final approval of manuscript: All authors. \\ \#These authors contributed equally to this work. \\ Correspondence to: Xiaomei Guo. Department of Cardiology, Tongji Hospital, Tongji Medical College, Huazhong University of Science and \\ Technology, Wuhan 430030, China. Email: xiaomguo@yeah.net.
}

Background: Heart-protecting musk pill (HMP), derived from Chinese herbal medicines, has been found to possess protective roles against atherosclerosis-related cardiovascular diseases (CVDs), however, the antiatherosclerotic mechanisms of HMP are still unclear. Here, we investigated the effects of HMP on alleviating atherosclerotic lesion severity in mice and explored the molecular mechanisms.

Methods: Apolipoprotein E-deficient mice were fed western-type diet supplemented with HMP (25 mg/kg/day) or normal saline gavage for 20 weeks. Then histopathological staining was performed to assess the atheromatous plaque burden. Biochemical kits were used to detect levels of lipid profiles. Moreover, effector factors associated with lipid metabolism in liver and intestinal tissues were investigated by western blot and real-time PCR assays. Levels of signal molecules participating in the mitochondrial-mediated apoptosis pathway were detected by Western blot.

Results: We found that HMP notably reduced atherosclerotic lesion size $(\mathrm{P}<0.05)$ and improved plaque stability $(\mathrm{P}<0.05)$. HMP treatment decreased circulating TC $(\mathrm{P}<0.01)$, LDL-C $(\mathrm{P}<0.01)$ and $\mathrm{TG}(\mathrm{P}<0.05)$ levels and increased HDL-C $(\mathrm{P}<0.05)$ content. HMP was found to suppress SREBP2, HMGCR and PCSK9 expressions $(\mathrm{P}<0.05)$, yet promote LDLR expression $(\mathrm{P}<0.05)$ in hepatocytes. Moreover, HMP was discovered to activate PPAR $\alpha / C P T-1 A$ cascade $(\mathrm{P}<0.05)$ and inhibit contents of SREBP1c and the lipogenic genes FAS and ACC $\alpha(\mathrm{P}<0.05)$. The LBK1/AMPK cascade was also activated after HMP administration $(\mathrm{P}<0.05)$. Additionally, HMP was found to facilitate transintestinal cholesterol excretion by increasing ABCG5 and ABCG8 levels and reducing NPC1L1 content $(\mathrm{P}<0.05)$. In terms of vasoprotective activities, we observed that HMP decreased cleaved caspase-3 content $(\mathrm{P}<0.05)$ in the vascular intima, which might be due to inhibition of mitochondrial-related signaling pathway.

Conclusions: Altogether, our study indicates that HMP plays anti-atherosclerotic roles via regulating lipid metabolism and improving vascular intimal injury.

Keywords: Heart-protecting musk pill (HMP); atherosclerosis; lipid metabolism; vascular intimal injury

Submitted Sep 04, 2019. Accepted for publication Nov 15, 2019.

doi: $10.21037 /$ atm.2019.12.22

View this article at: http://dx.doi.org/10.21037/atm.2019.12.22 


\section{Introduction}

Atherosclerosis, featured by progressive plaque lesion formation leading to lumen stenosis commonly occurring in large and medium-sized artery walls, is the principal pathological basis of cardiovascular diseases (CVDs) (1). The development of atheroma lesions is associated with a series of chronic processes involving lipid metabolic dysfunction and vascular intimal injury $(2,3)$. Damaged intima facilitates excessive lipid deposition and circulating leukocytes accumulation in the vessel subendothelial space, which are pivotal pathogenic events facilitating atherogenesis (4). It is well-established that dyslipidemia is a crucial risk factor for atherosclerosis and regulation of blood lipid profiles has been given priority in the treatment of atherosclerotic CVDs $(2,5)$. Moreover, therapeutic approaches protecting against vascular intimal impairment have been proved to inhibit atheroma lesion progression $(6,7)$. Currently, several modern drugs such as statins have been used for the treatment of atherosclerosis. However, up to $20 \%$ patients are unable to tolerate statin treatment due to the side effects and the residual risk of adverse cardiovascular events is still high in several patients treated with statins $(8,9)$. Accordingly, highly safe and effective agents targeting the atherogenic processes are urgently needed.

Heart-protecting musk pill (HMP), deriving from an ancient traditional Chinese medicine (TCM) Suhexiang Pill, possesses therapeutic properties against several CVDs in clinical application, such as ischemic heart disease, heart failure and hypertension (10). HMP comprises seven medicinal substances: Radix Ginseng (Panax ginseng C.A.Mey. root), Venenum Bufonis, Styrax (Liquidambar orientalis Mill. balsam), Calculus Bovis Artifactus, Cortex Cinnamomi (Cinnamomum cassia Presl. bark), Borneolum Syntheticum (Dryobalanops aromatica C.F.Gaertn. resin) and Artificial Moschus (11). Zhao et al. have indicated that HMP is capable of alleviating hypertensive renal injury by suppressing the TLR4/NF- $\mathrm{BB}$ inflammatory pathway (11). Moreover, HMP has been found to be a potent modulator of blood lipid spectrum in the hyperlipidemic state (12). It has been observed that HMP improves cardiac function and reduces damaged area in rats with myocardial infarction via promoting endothelial progenitor cell mobilization and proliferation (13). In addition, findings of other studies show that HMP is capable of alleviating cardiac pathological remodeling through modulating signal transduction of TGF- $\beta /$ Smads pathway (14). Recently, there is evidence showing that HMP plays roles in inhibiting atherosclerosis development $(12,15)$. However, the exact anti-atherosclerotic mechanisms of HMP are not fully understood. Here, we investigated the atheroprotective effects of HMP using an animal model of atherosclerosis induced by western-type diet (WTD) and determined the potential underlying mechanisms of action.

\section{Methods}

\section{Animal procedure}

Adult 24 male homozygous apolipoprotein E knockout $\left(\mathrm{apoE}^{-/}\right)$mice at 8 weeks with C57BL/6 background were purchased from Vital River Laboratory Animal Technology Co., Ltd. (Beijing, China). Before the experiments, mice were acclimatized for 2 weeks and housed separately in individually ventilated cages. All animals were maintained in a controlled temperature $\left(22 \pm 2{ }^{\circ} \mathrm{C}\right)$ under a 12 -h light/ dark cycle with ad libitum access to standard food and water. Then, apoE $\mathrm{E}^{-/-}$mice at 10 weeks old were fed a WTD (containing $21 \%$ fat and $0.15 \%$ cholesterol) and randomly allocated into two groups ( $\mathrm{n}=12$ each group). One group (HMP) was administered $25 \mathrm{mg} / \mathrm{kg}$ /day HMP (provided by Shanghai Hutchison Pharmaceuticals, Shanghai, China, batch number 181107) suspended in normal saline intragastrically $(13,16,17)$. Another group (WTD) was intragastrically treated with the same volume of normal saline per day. After 20 weeks of drug delivery, all mice were fasted overnight and euthanized by injecting pentobarbital sodium intraperitoneally (Figure 1).

Blood samples were acquired from the orbital veins and centrifuged at $3,000 \mathrm{rpm}$ at $4{ }^{\circ} \mathrm{C}$ for $10 \mathrm{~min}$. The top serum layer was collected and stored at $-80{ }^{\circ} \mathrm{C}$ until use. The residual blood in solid tissues was removed by wholebody perfusion with ice-cold $0.9 \%$ normal saline through the left ventricle. The liver, intestine, heart and aorta tissues were then harvested for subsequent experiments. All animal procedures were approved by the Institutional Animal Care and Use Committee of Tongji Medical College, Huazhong University Science and Technology, Wuhan, China. All experiment procedures conformed to the National Institutes of Health Guide for the Care and Use of Laboratory Animals.

\section{Characterization of aortic atherosclerotic plaques}

The full-length aorta from the root to the common iliac 


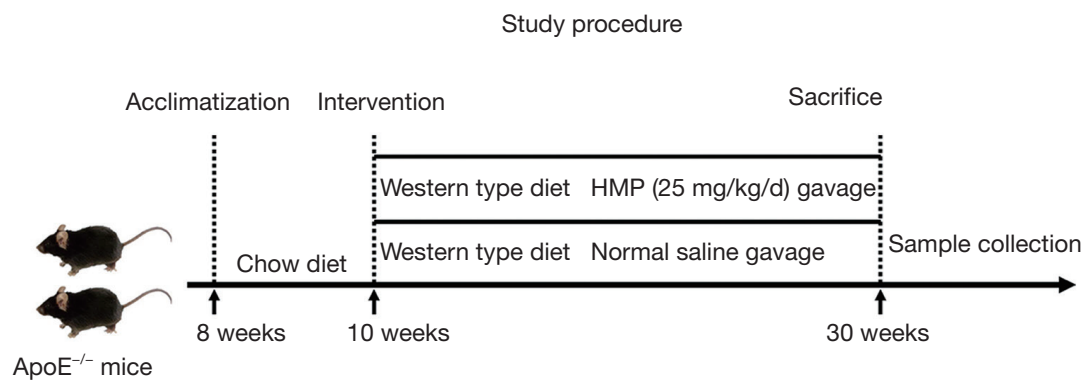

Figure 1 The schematic experimental procedure of this study. HMP, heart-protecting musk pill.

artery was rapidly removed and the extent of atherosclerotic lesions in the whole aorta lumen surface was assessed by Oil Red O staining (Sigma, St. Louis, USA). En face of the entire aorta was photographed and the atheroma plaque area was measured using an image analysis program (Image-pro plus 6.0 software, Media Cybernetics, USA). Atherosclerosis severity was determined as the plaque lesion size relative to the inner surface area of the total aorta.

The aorta segments were fixed in $4 \%$ paraformaldehyde solution and embedded in paraffin. Afterwards, the aorta tissues were serially cut into 5 - $\mu \mathrm{m}$ thick cross-sections at $50-\mu \mathrm{m}$ intervals. A set of paraffin sections were stained with hematoxylin and eosin (H\&E) for morphological analysis of the atherosclerotic lesions. Subsequently, the collagen content within the plaque area was visualized by Masson's trichrome staining. Moreover, immunohistochemical detection of cross-sections was performed to quantify the levels of monocyte macrophages 2 (MOMA-2), $\alpha$ smooth muscle actin ( $\alpha$-SMA) (Abcam, Cambridge, UK) and cleaved caspase-3 (Cell Signaling Technology, MA, USA). Quantitative analysis of the indicators was performed with the Image-pro plus 6.0 software.

\section{Histomorphometric evaluation of liver and intestine}

Liver and intestine tissues were excised and fixed in $4 \%$ paraformaldehyde solution. The tissues were then dehydrated and embedded in paraffin. Serial 5 - $\mu \mathrm{m}$ thick cross-sections were harvested and stained with $\mathrm{H} \& \mathrm{E}$ following standard procedures.

The liver tissue samples were embedded in optimal cutting temperature compound and sectioned into 8- $\mu \mathrm{m}$ consecutive sections using a cryostat. The sections were stained with Oil Red $\mathrm{O}$ for the evaluation of lipid content in liver tissues.

\section{Detection of lipid profiles}

Serum concentrations of triglyceride (TG), total cholesterol (TC), low density lipoprotein cholesterol (LDL-C) and high-density lipoprotein cholesterol (HDL-C) were determined with commercially available reagents according to the manufacturer's protocols (Jiancheng Bioengineering Institute, Nanjing, China).

For quantifying hepatic and fecal lipid levels, the liver tissue and fecal particles were homogenized with ethyl alcohol. The homogenate was then centrifuged and the top liquid was collected for measuring TG and TC contents using biochemical kits (Jiancheng Bioengineering Institute, Nanjing, China).

\section{Quantification of circulating bioactive factors}

Concentrations of inflammatory cytokines in the serum including TNF- $\alpha$, IL-1 $\beta$, IL-6, and CRP were quantified using the ELISA kits (Boster Biological Technology, Wuhan, China). Moreover, the circulating NO content was evaluated by biochemical kits (Jiancheng Bioengineering Institute, Nanjing, China). Briefly, $100 \mu \mathrm{L}$ of serum sample was mixed uniformly with $300 \mu \mathrm{L}$ of pre-processing reagent using a vortex finder. Then the mixture was centrifuged at 4,000 rpm for $15 \mathrm{~min}$ at room temperature and the supernatant was collected. Afterwards, $160 \mu \mathrm{L}$ of the obtained supernatant was incubated with $80 \mu \mathrm{L}$ of the chromogenic agent for $15 \mathrm{~min}$. The absorbance was detected at $560 \mathrm{~nm}$ on a microplate reader.

\section{Western blot assay}

For protein extraction, the liver, intestine and aorta tissues were lysed with RIPA lysis buffer supplemented with protease and phosphatase inhibitors. The concentrations 
Table 1 The nucleotide sequence of the sense and antisense primers used in real-time quantitative PCR

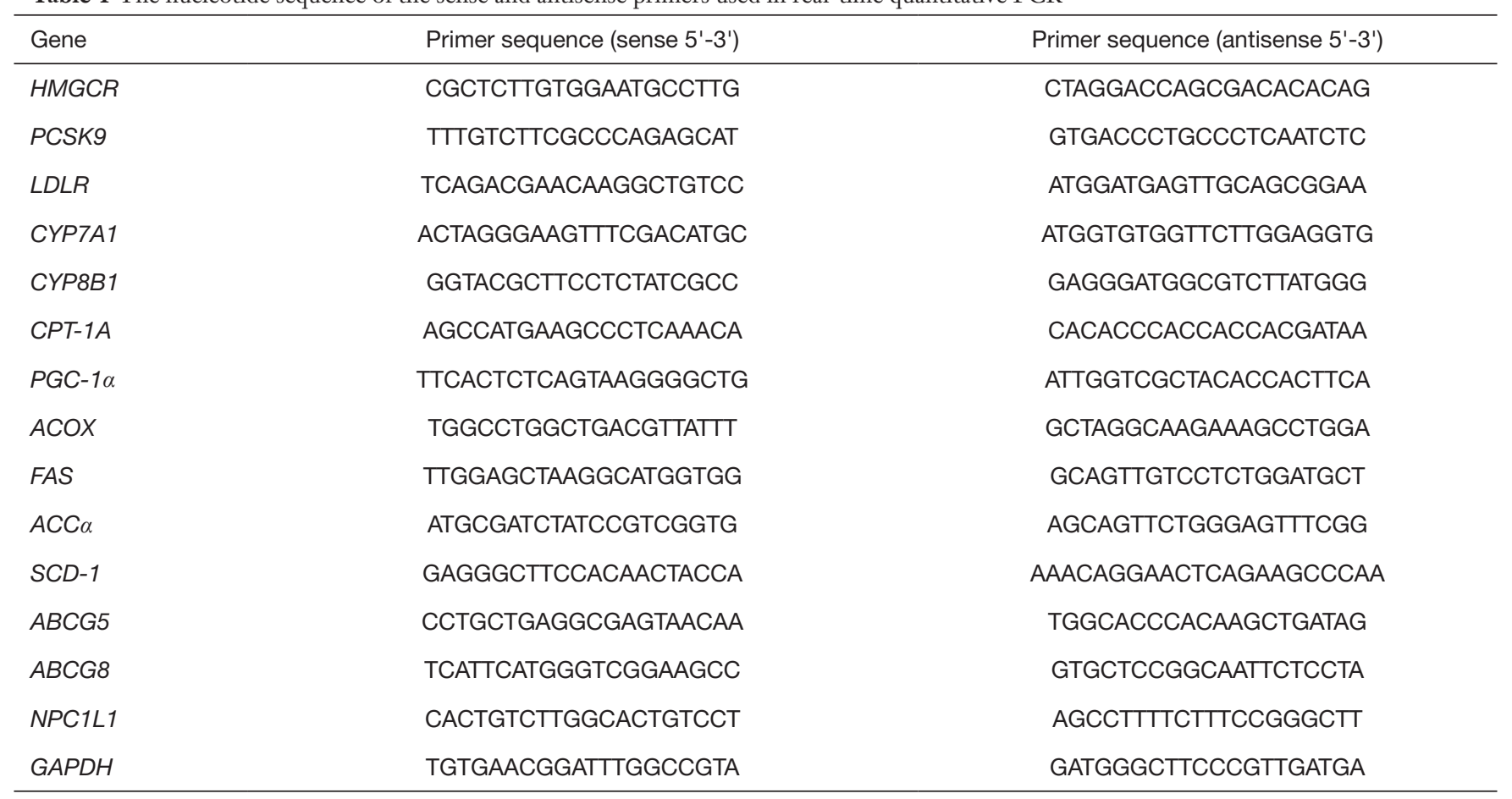

of extracted proteins were tested using bicinchoninic acid assay. After denaturation, proteins were loaded on $10 \%$ sodium dodecyl sulfate-polyacrylamide electrophoresis gel for separation. The separated proteins were then transferred onto polyvinylidene difluoride membranes, which were sequentially blocked with $5 \%$ BSA dissolved in TBS-T for $1 \mathrm{~h}$ at room temperature and probed with appropriate primary antibodies overnight at $4{ }^{\circ} \mathrm{C}$. The membranes were then washed with TBS-T and incubated with horseradish peroxidase-conjugated secondary antibodies at room temperature for $1 \mathrm{~h}$. Then protein bands were visualized using an enhanced chemiluminescence detection reagent and the acquired images were analyzed with the Image $\mathrm{J}$ software (NIH, USA). The primary antibodies against sterol regulatory element binding protein 2 (SREBP2), 3-hydroxy-3-methylglutarylcoenzyme A reductase (HMGCR), LDL receptor (LDLR), proprotein convertase subtilisin/kexin type 9 (PCSK9), peroxisome proliferator-activated receptor $\alpha(\mathrm{PPAR} \alpha)$ and SREBP1c were obtained from Abcam (Cambridge, UK). Antibodies against c-Raf, p-c-Raf, ERK1/2, p-ERK1/2, AMPK, p-AMPK, calcium/calmodulin-dependent kinase kinase $\beta(\mathrm{CaMKK} \beta)$, p-CaMKK $\beta, \mathrm{Bcl}-2, \mathrm{Bax}, \mathrm{Bad}$, Apaf-1, caspase-9 and caspase-3 were purchased from Cell Signaling Technology (MA, USA). Anti-liver kinase
B1 (LKB1), anti-p-LKB1, anti-Niemann-pick C1-like 1 (NPC1L1) and anti-Bcl-xl were obtained from Santa Cruz Biotechnology (CA, USA). Moreover, anti-p-HMGCR and anti-camitine palmitoyltransferase $1 \mathrm{~A}$ (CPT-1A) were obtained from Bioss (MA, USA). Anti-ATP-binding cassette transporter G5 (ABCG5) and anti-ABCG8 were offered by Affinity Biosciences (OH, USA). Antibodies against 7alpha-hydroxylase (CYP7A1), fatty acid synthase (FAS), acetyl-CoA carboxylase $\alpha(\mathrm{ACC} \alpha), \mathrm{p}-\mathrm{ACC} \alpha$, cytochrome-c and GAPDH were purchased from Abclonal (Boston, USA).

\section{Real-time quantitative PCR}

Total RNA was isolated from the frozen hepatic and intestinal tissues with RNAiso Plus (Takara, Japan). The concentration of extracted RNA was determined using a BeckMan Coulter DU730. Complementary DNAs were synthesized from total RNA templates using a PrimeScript ${ }^{\mathrm{TM}}$ RT reagent (Takara, Japan). Then mRNA expression level of each gene was detected by quantitative real-time PCR using TB Green kits (Takara, Japan). mRNA contents of target molecules were normalized to that of GAPDH, which was selected as an internal standard. Primers used here were listed in Table 1. 


\section{Statistical analysis}

All data were presented as the mean \pm standard deviation and data analysis was performed using the SPSS software version 21.0. The Kolmogorov-Smirnov test and Levene test was used to analyze the normality and homogeneity of variances of data, respectively. Student's $t$-test was applied to compare the significance of differences between two groups. $\mathrm{P}$ values less than 0.05 (two-tailed) were considered to be statistically significant.

\section{Results}

\section{HMP ameliorated the severity of atherosclerotic lesions}

The lesion area in the aortic inner surface was quantified by Oil Red O staining and we observed that the atherosclerotic lesion range in three aortic segments of HMP-treated mice was dramatically decreased in comparison with those of the WTD mice $(\mathrm{P}<0.05$ for aortic arch, $\mathrm{P}<0.05$ for thoracic aorta, $\mathrm{P}<0.01$ for abdominal aorta) (Figure $2 A$ ). Moreover, the analysis of $\mathrm{H} \& \mathrm{E}-\mathrm{stained}$ aortic cross-sections revealed that HMP administration effectively alleviated the atherosclerotic burden, as seen by decrease of the percentage of plaque size to total aortic lumen area $(\mathrm{P}<0.05)$ and lesion thickness reduction at the site of atheromatous plaques $(\mathrm{P}<0.05)$ (Figure $2 B)$.

We then assessed the effects of HMP on regulating the stability of atherosclerotic lesions. Histopathological detection showed that the plaque lesion in mice with HMP gavage possessed smaller necrotic core $(\mathrm{P}<0.01)$ and higher collagen content $(\mathrm{P}<0.01)$ compared with vehicletreated mice, as indicated by H\&E staining and Masson' trichrome staining (Figure 3A,B). Immunohistochemical test discovered that the lesion area from HMP-treated samples had reduced expression of MOMA-2 $(\mathrm{P}<0.05)$, which was a specific marker for macrophages, suggesting the decrease of macrophage accumulation in aortic plaques (Figure 3C). Additionally, the content of smooth muscle cell was increased in the plaques of HMP-treated mice $(\mathrm{P}<0.05)$, as assessed by $\alpha$-SMA staining (Figure $3 D$ ). In view of above findings, HMP was proved to encumber atherosclerosis progression and strengthen the stability of plaque lesions.

\section{HMP displayed effective effects on mediation of serum lipid spectrum}

To analyze the mechanisms by which HMP reduced atherosclerosis progression, we first examined the effect of
HMP on blood lipid profiles, given that dyslipidemia was an early pathological event responsible for atherogenesis (2). As showed in Figure 4A, HMP treatment lowered TG $(\mathrm{P}<0.05)$, TC $(\mathrm{P}<0.01)$ and LDL-C $(\mathrm{P}<0.01)$ contents, while elevating serum HDL-C level $(\mathrm{P}<0.05)$ when compared with normal saline management.

\section{HMP markedly mitigated the extent of lipid accumulation in the liver}

We then evaluated the effects of HMP on WTD-induced lipid disposition in the liver. Oil Red O staining of liver cryosections showed that numerous lipid droplets existed in liver tissues of the WTD-fed mice and HMP intervention significantly decreased the number and size of lipid droplets in hepatic tissues. Moreover, the cytoplasm of hepatocytes from vehicle-treated mice exhibited many fat vacuoles, as evidenced by $\mathrm{H} \& \mathrm{E}$ staining. We discovered that the severity of vesicular steatosis of hepatocytes was alleviated in the HMP-treated mice (Figure $4 B$ ). Similar to histological detection results, TG $(\mathrm{P}<0.05)$ and $\mathrm{TC}(\mathrm{P}<0.05)$ contents in liver tissues were dramatically decreased in the HMPtreated mice when compared to those in the WTD-fed mice (Figure 4C).

\section{HMP regulated signal molecules related with lipid metabolism in hepatic tissues}

Since the liver was a pivotal organ involved in lipid metabolism (18), we evaluated whether HMP improved lipid profiles via regulating relevant signal molecules in the liver of $\mathrm{apoE}^{-/-}$mice. We observed that HMP administration diminished the expressions of SREBP2 $(\mathrm{P}<0.01)$ and downstream molecules including HMGCR $(\mathrm{P}<0.05)$ and PCSK9 $(\mathrm{P}<0.05)$, which regulated cholesterol synthesis and LDLR degradation, respectively. LDLR responsible for LDL-C uptake by hepatocytes was another SREBP2modulated downstream effector (19). However, LDLR was found to be upregulated in hepatocytes of the HMP group $(\mathrm{P}<0.05)$ (Figure $5 A)$. We further analyzed the Raf/ERK1/2 pathway which regulated $L D L R$ mRNA stability (20). We found that HMP treatment enhanced the activities of Raf and ERK1/2 $(\mathrm{P}<0.05)$ (Figure $5 B)$, hinting that HMP might enhance the stability of $L D L R$ mRNA. Moreover, no difference in CYP7A1 level $(\mathrm{P}>0.05)$, which regulated cholesterol bioconversion, was found in liver tissues between the two groups (Figure 5C). PPAR $\alpha / C P T-1 \mathrm{~A}$ pathway was reported to facilitate $\beta$-oxidation of fatty acids (21). Whereas, 

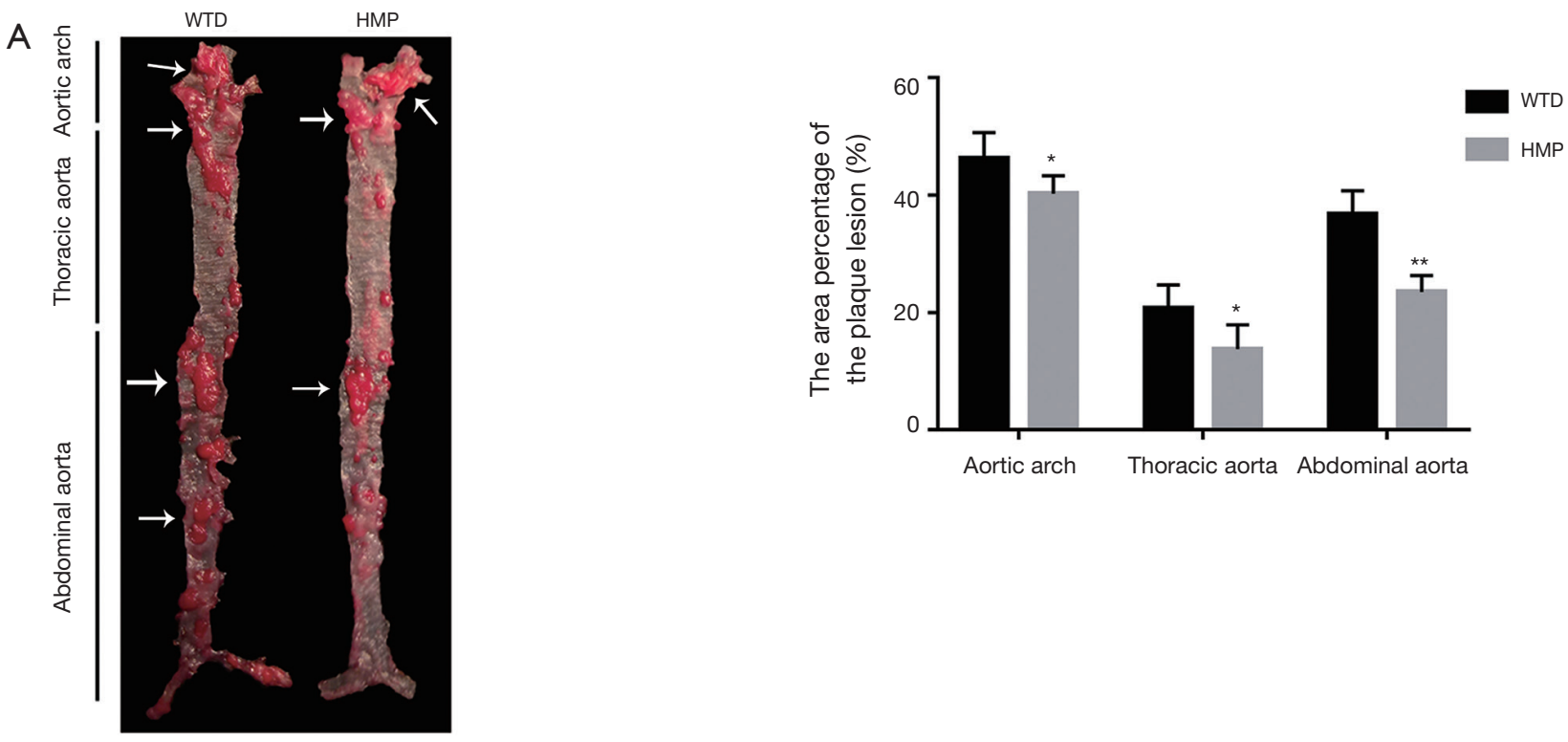

B

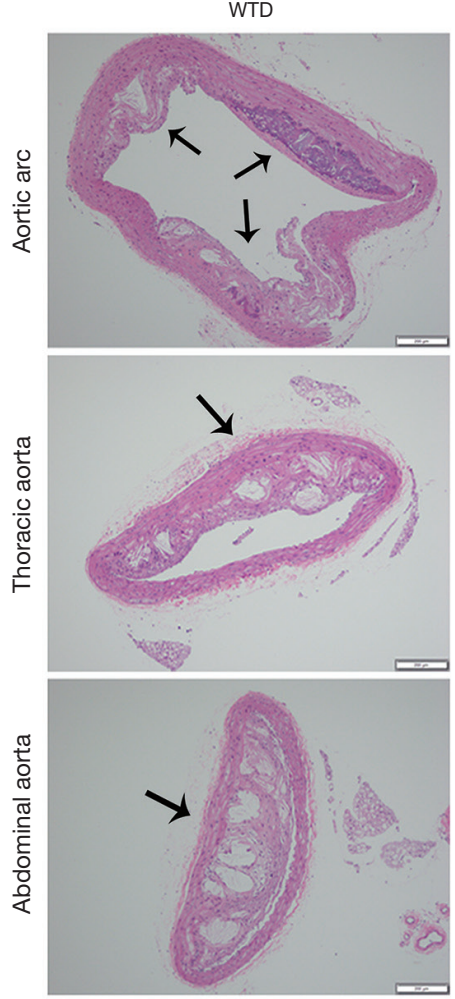

HMP
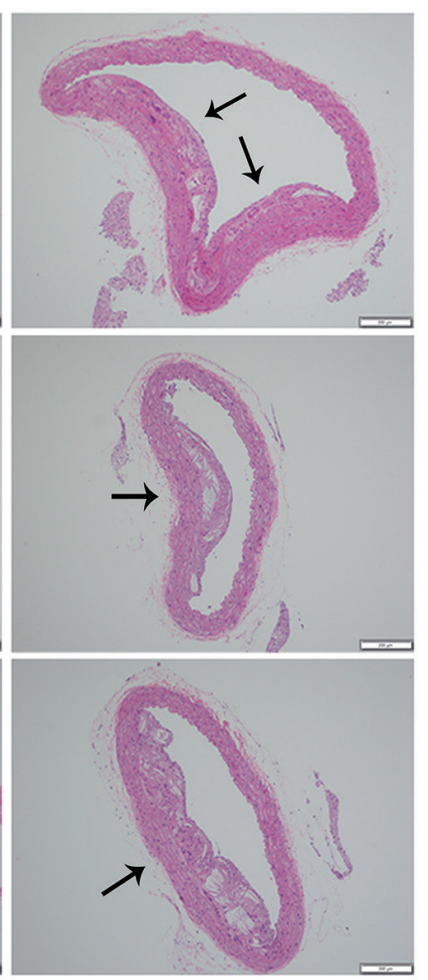

60
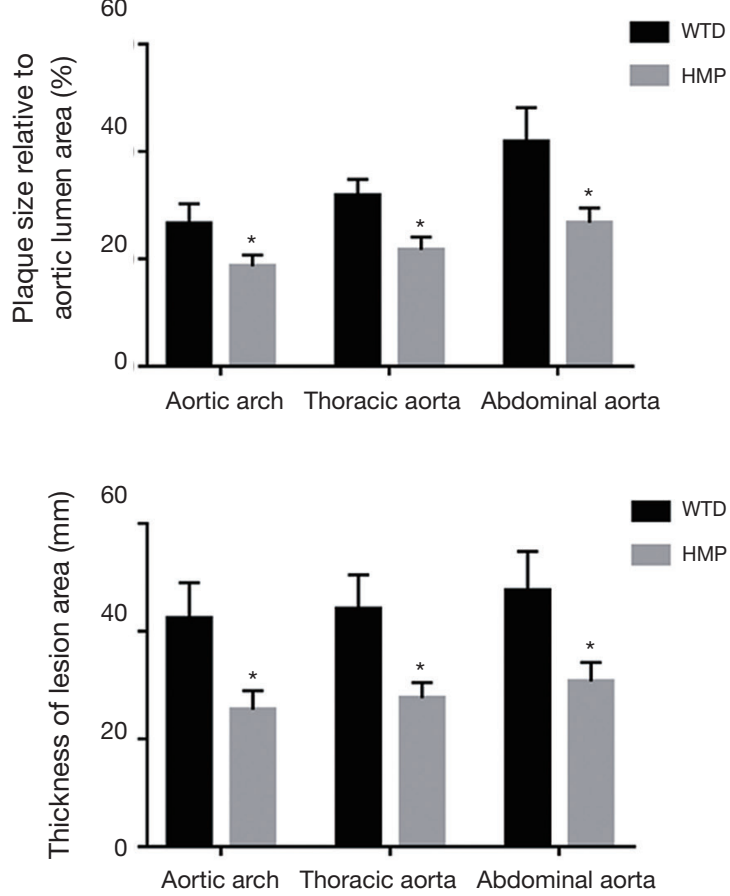

Figure 2 Heart-protecting musk pill (HMP) administration attenuated the progression of atherosclerosis in $\mathrm{apoE}^{-/-}$mice. (A) Representative images of plaque lesions in the area of aortic inner surface stained with Oil Red O. The arrows indicated the plaques in the aortic inner surface. (B) H\&E staining assessed the extent of plaque-induced luminal stenosis and the degree of lesion hyperplasia in three segments of the entire aorta. The arrows showed the plaques in the cross-section. Data were expressed as the mean $\pm \mathrm{SD}, \mathrm{n}=5 .{ }^{*} \mathrm{P}<0.05,{ }^{* *} \mathrm{P}<0.01$ vs. WTD group. Scale bar $=200 \mu \mathrm{m}$. 
A

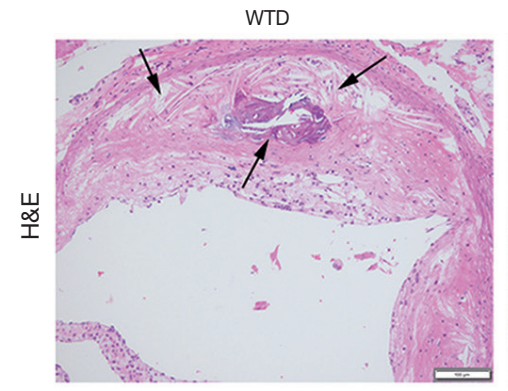

B

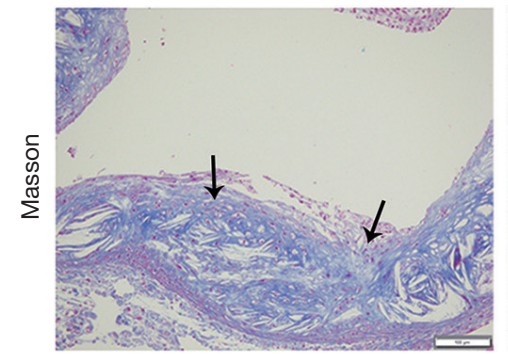

C

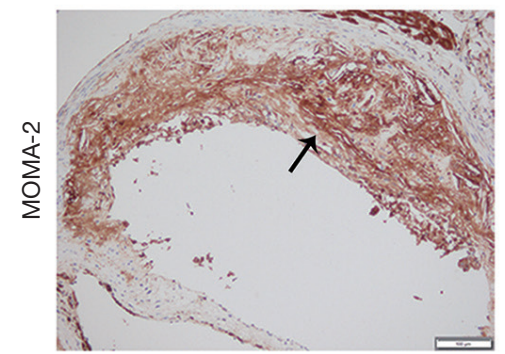

D

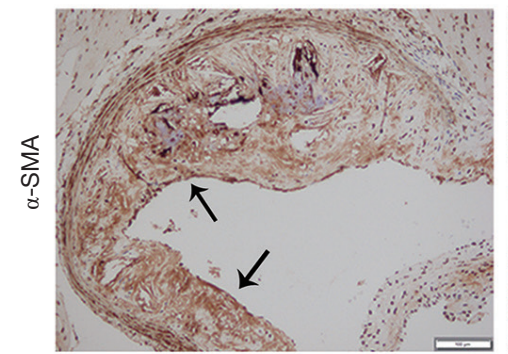

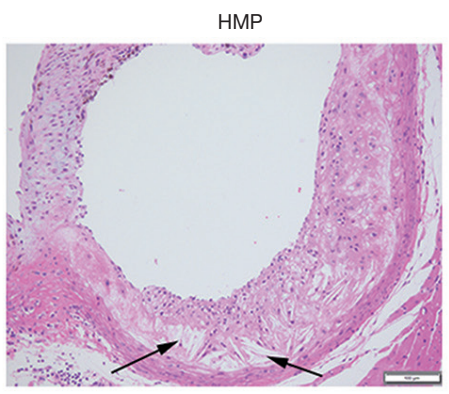
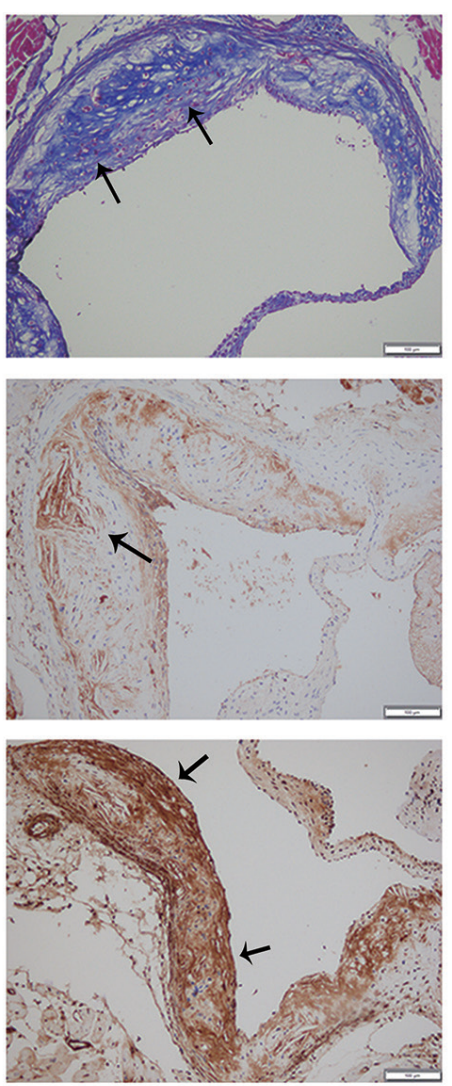
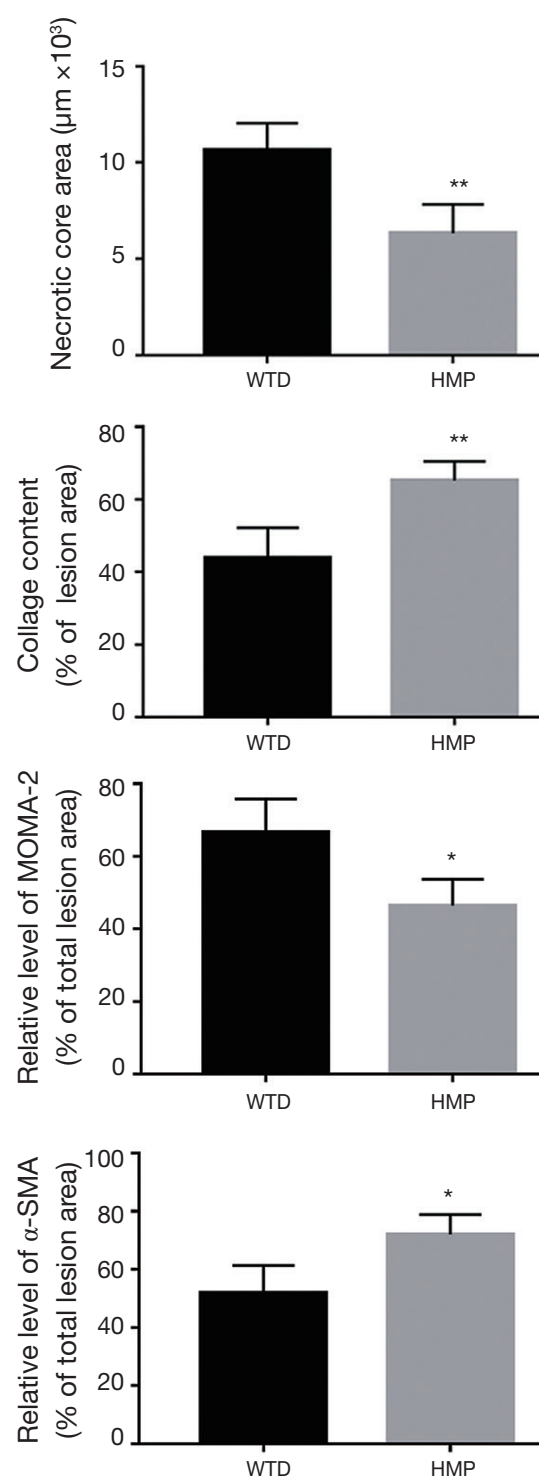

Figure 3 The effects of heart-protecting musk pill (HMP) on the stability of atherosclerotic lesions in the aortic root. (A) The size of necrotic core in the plaque area was determined by H\&E staining. The arrows showed the necrotic core in the plaques. (B) The collagen content was detected with Masson's Trichrome staining. The arrows indicated the area of collagen. (D) Immunostaining for MOMA-2 (C) and $\alpha$-SMA (D) in the lesion area. The arrows indicated the representative area. Results were presented as the mean $\pm \mathrm{SD}, \mathrm{n}=5 .{ }^{*} \mathrm{P}<0.05$, ${ }^{* *} \mathrm{P}<0.01$ vs. WTD group. Scale bar $=200 \mu \mathrm{m}$.

SREBP1c and its target molecules FAS and ACC $\alpha$ were key factors involved in de novo lipogenesis $(19,22)$. Here, we discovered that HMP might trigger TG catabolism and weaken TG anabolism, as seen by level elevation of PPAR $\alpha$ $(\mathrm{P}<0.01)$ and $\mathrm{CPT}-1 \mathrm{~A}(\mathrm{P}<0.05)$ and content decrement of SREBP1c, FAS and ACC $\alpha(\mathrm{P}<0.05)$ (Figure $5 D, E)$.

Furthermore, AMPK, which mediated the up-regulation of PPAR $\alpha$ and down-regulation of SREBPs (23), was found to present enhanced activities in hepatic tissues of HMP-treated mice $(\mathrm{P}<0.05)$ (Figure $5 F)$. HMGCR and ACC, acting as the phosphorylation substrates of AMPK, presented a noticeable elevation in the phosphorylated level of the HMP group $(\mathrm{P}<0.05)$ (Figure $5 A, E)$. Moreover, we observed that HMP strengthened the activity of LKB1 

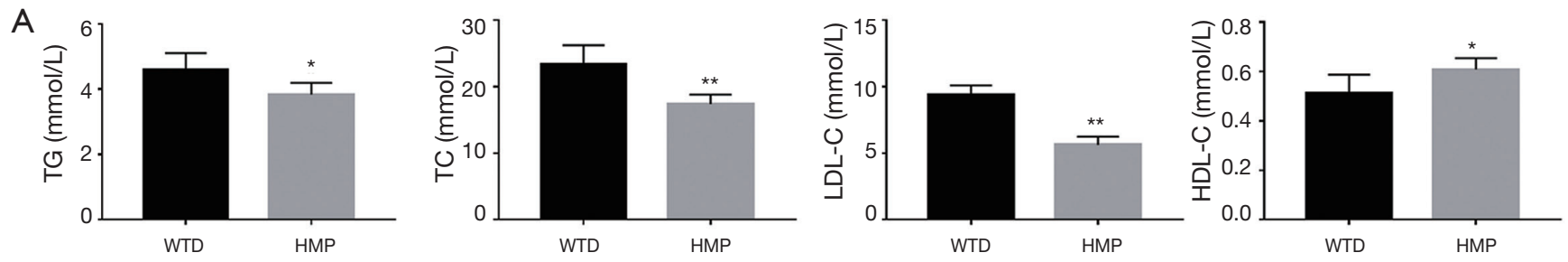

B
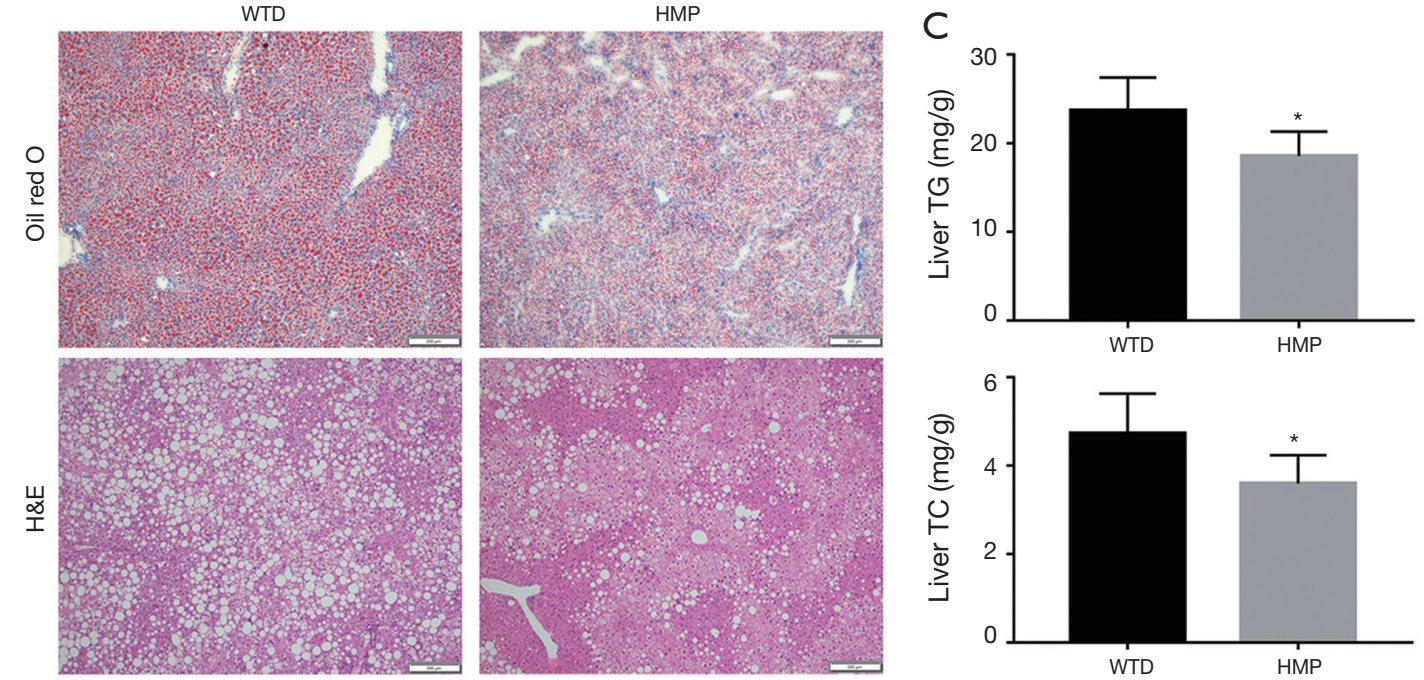

Figure 4 Heart-protecting musk pill (HMP) treatment played effective roles in the regulation of lipid profiles in the bloodstream and the liver. (A) The concentrations of serum TG, TC, LDL-C and HDL-C. (B) Representative micrographs of liver sections stained with H\&E and Oil Red O, respectively. (C) The contents of liver TG and TC. Data were expressed as the mean $\pm \mathrm{SD}, \mathrm{n}=6$. ${ }^{*} \mathrm{P}<0.05$, ${ }^{* *} \mathrm{P}<0.01$ vs. WTD group. Scale bar $=200 \mu \mathrm{m}$.

responsible for AMPK activation $(\mathrm{P}<0.05)$, yet had no impact on the activity of CaMKK $\beta(\mathrm{P}>0.05)$, which was another upstream AMPK kinase (Figure $5 F)$.

As shown in Figure 5G, HMP reduced HMGCR and PCSK9 mRNA content and raised LDLR mRNA level $(\mathrm{P}<0.05)$. Meanwhile, compared with the WTD mice, mRNA expressions of $F A S$ and $A C C \alpha(\mathrm{P}<0.05)$ were decreased and levels of $C P T-1 A$ and peroxisome proliferatoractivated receptor gamma coactivator $1-\alpha(P G C-1 \alpha) \mathrm{mRNA}$ $(\mathrm{P}<0.05)$ were increased in liver tissues of HMPadministered mice. In addition, there were no significant differences in levels of CYP7A1, sterol-12 $\alpha$-bydroxylase (CYP8B1), acyl CoA oxidase $(A C O X)$ and stearoyl-CoA desaturase $1(S C D-1)$ mRNA $(\mathrm{P}>0.05)$ between the two groups.

\section{HMP affected levels of factors involved in lipid transportation in intestinal tissues}

Intestine was found to affect blood lipid profiles via regulating lipid absorption and excretion (24). We found that HMP raised TC content $(\mathrm{P}<0.05)$ but did not affect TC level $(\mathrm{P}>0.05)$ in feces of apoE $\mathrm{E}^{-/-}$mice (Figure $\left.6 A\right)$. Additionally, no significant differences in food intake and excrement weight were observed between the two groups $(\mathrm{P}>0.05)$ (Figure 6B). H\&E staining revealed that HMP oral gavage did not alter the architecture and morphology of intestinal tissues (Figure 6C). Then, we discovered that HMP treatment increased the expression of cholesterol transporter $A B C G 5$ and $A B C G 8$, yet reduced both NPC1L1 mRNA and protein levels $(\mathrm{P}<0.05)$ (Figure $6 D, E)$, implying that HMP probably elevated fecal TC content via mediating transintestinal cholesterol migration.

\section{HMP decreased levels of circulating inflammation- promoting cytokines}

Since inflammation was a vital contributor to atherosclerosis initiation and progression by inducing vascular endothelial injury $(25,26)$, we hypothesized that HMP improved the 


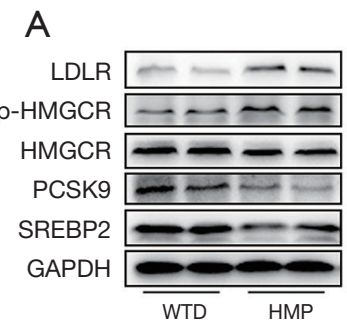

C
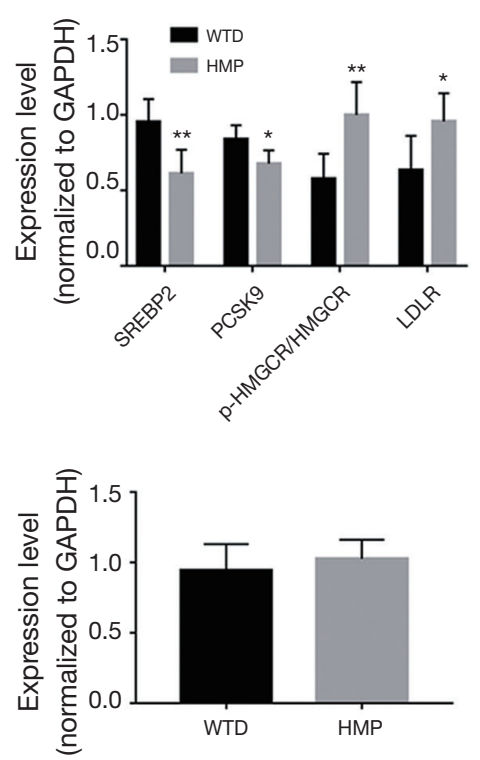

$\mathrm{E}$

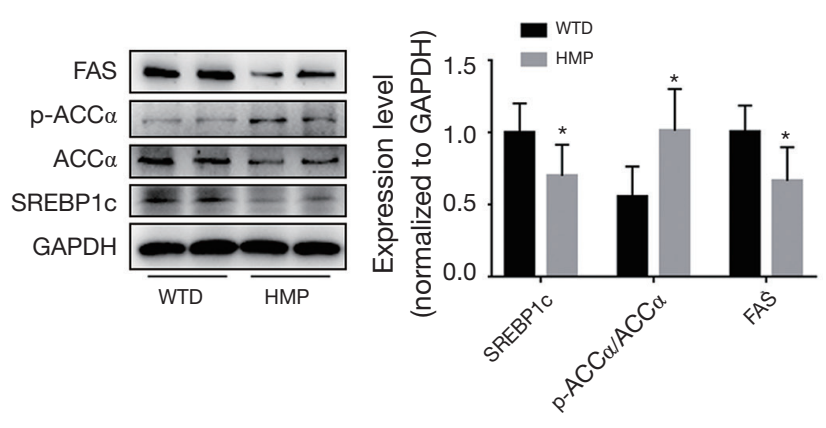

G

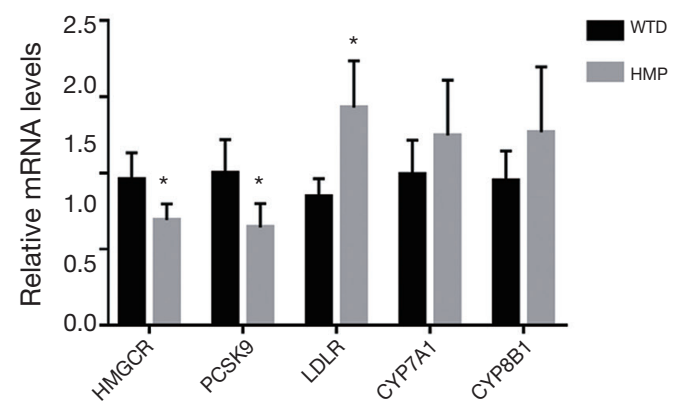

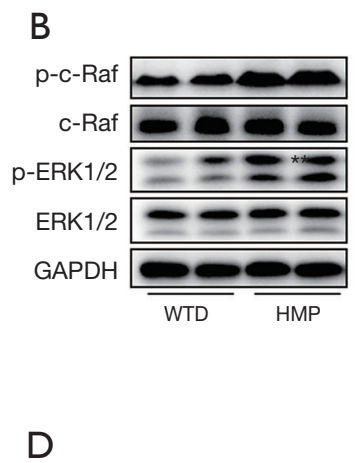
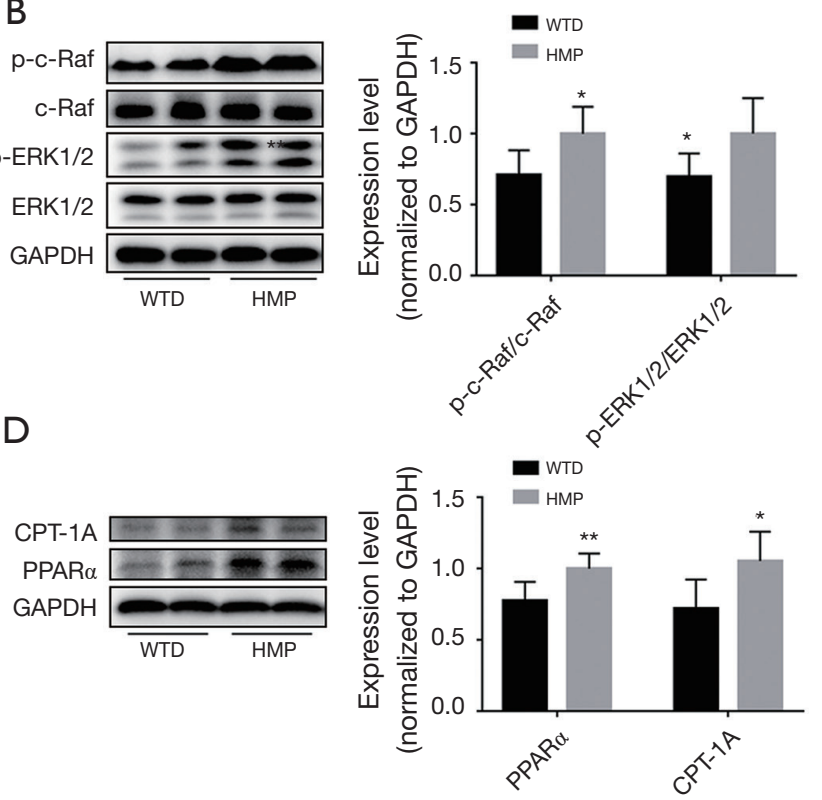

$\mathrm{F}$
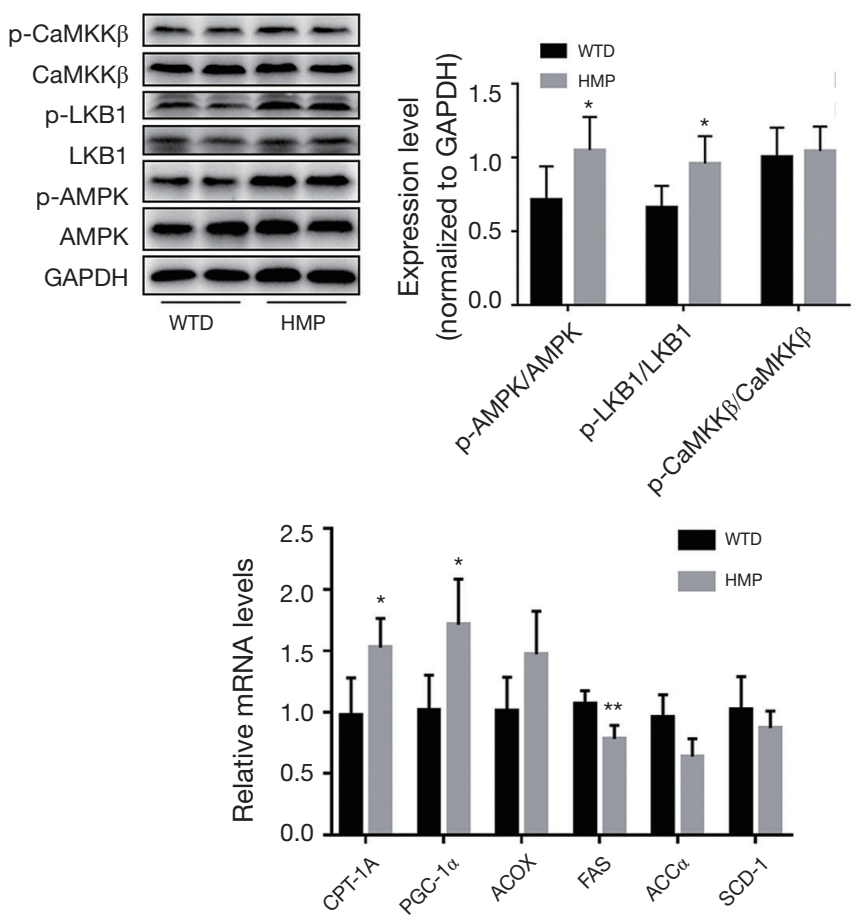

Figure 5 Heart-protecting musk pill (HMP) affected the expressions and activities of molecules participating in hepatic lipid metabolism. (A) Protein levels of SREBP2 and downstream effectors involved in cholesterol biosynthesis and uptake. (B) Signal transduction of Raf/ERK1/2 pathway was examined by western blot. The protein expression of CYP7A1 (C), PPAR $\alpha$ and CPT-1A (D). (E) Protein levels of SREBP1c and downstream factors involved in de novo lipogenesis. (F) Total protein content and corresponding phosphorylated level of AMPK, LKB1 and CaMKK $\beta$. (G) The mRNA expressions of effector genes associated with cholesterol (left part) and fatty acid (right part) metabolism. Results were presented as the mean $\pm \mathrm{SD}, \mathrm{n}=6 .{ }^{*} \mathrm{P}<0.05,{ }^{* *} \mathrm{P}<0.01$ vs. WTD group. 


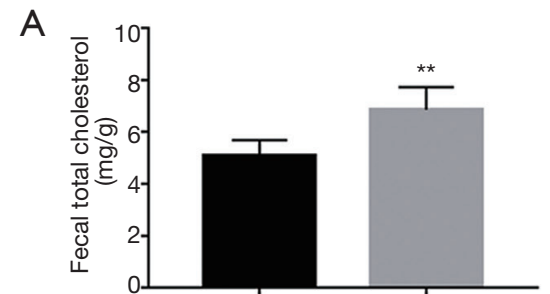

B

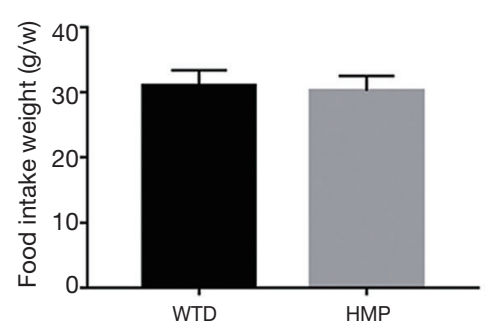

C

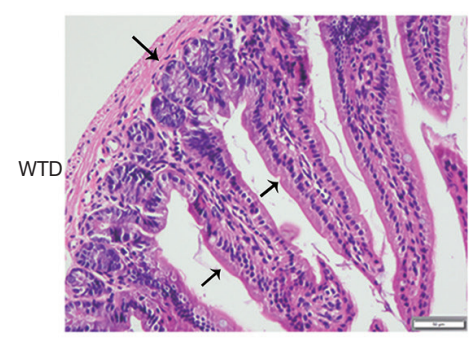

$\mathrm{E}$

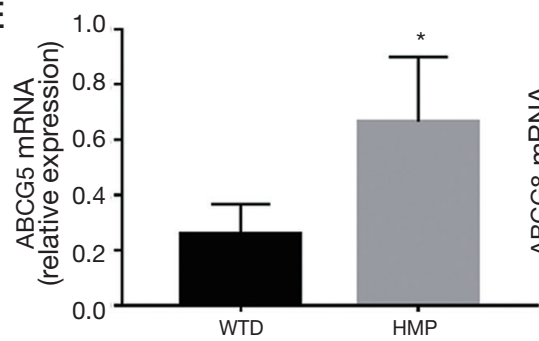

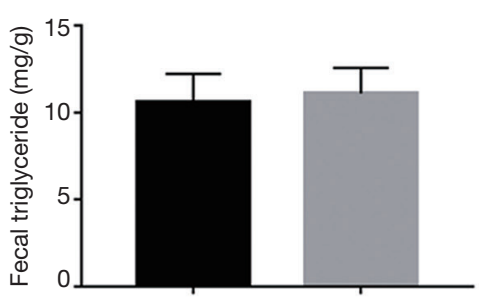
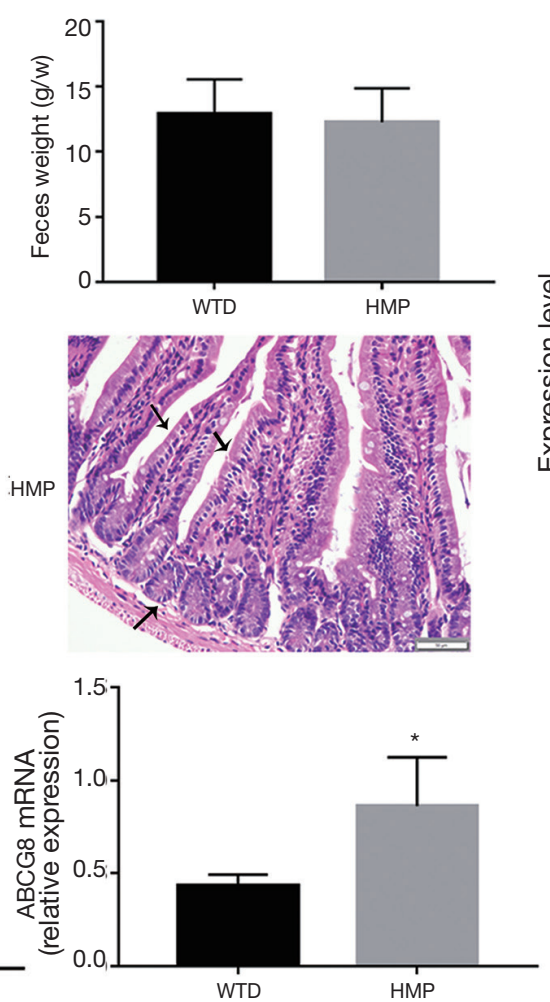

D
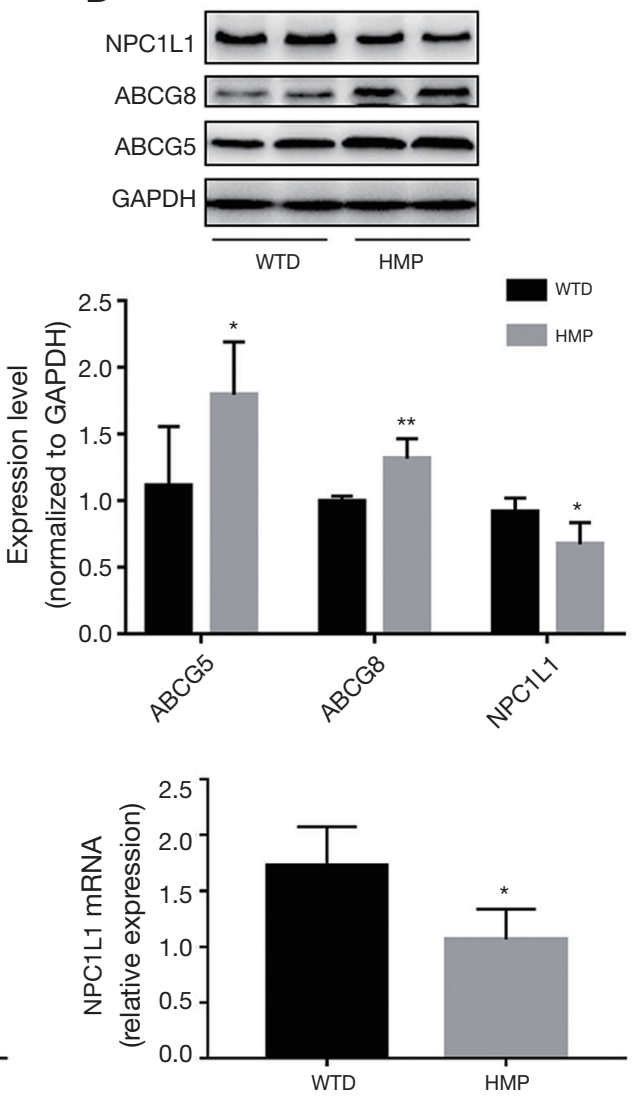

Figure 6 The regulatory roles of heart-protecting musk pill (HMP) in intestinal lipid metabolism. (A) The contents of TC and TG in the fecal particles. (B) The food intake and feces weight of apo $\mathrm{E}^{-/-}$mice per week. (C) Representative images of intestine sections stained with H\&E. The arrows showed the features of the intestine tissue. The protein (D) and mRNA (E) levels of ABCG5, ABCG8 and NPC1L1. Data were expressed as the mean $\pm \mathrm{SD}, \mathrm{n}=6 .{ }^{*} \mathrm{P}<0.05,{ }^{* *} \mathrm{P}<0.01$ vs. WTD group. Scale bar $=50 \mu \mathrm{m}$.

hyper-inflammatory state in vivo. To validate this hypothesis, serum cytokine levels were quantified. We found that levels of serum TNF- $\alpha$, IL-1 $\beta$, IL-6 and CRP were dramatically decreased in response to HMP intervention $(\mathrm{P}<0.05)$ (Figure $7 A$ ).

\section{HMP had beneficial roles against vascular intimal injury}

As vascular intimal damage augmented extension of atheroma plaques (27), we assessed the impact of HMP on tunica intima injury. As shown in Figure $7 B$, there was a content decrement of cleaved caspase- 3 in the arterial intima of HMP-administered mice $(\mathrm{P}<0.05)$. Then, results of western blot suggested that HMP raised the $\mathrm{Bcl}-2 / \mathrm{Bax}$ ratio and $\mathrm{Bcl}-\mathrm{xl}$ level $(\mathrm{P}<0.01)$ and reduced $\mathrm{Bad}$ content $(\mathrm{P}<0.05)$ in aortic tissues (Figure 7C). The cytoplasm cytochrome-c, necessary for apoptotic signal transduction, was found to exhibit a lowered content after HMP treatment $(\mathrm{P}<0.01)$. Meanwhile, HMP reduced levels of Apaf- 1 and decreased contents of cleaved caspase- 9 and cleaved caspase- $3(\mathrm{P}<0.05)$ (Figure $7 D$ ). These findings suggested that HMP effectively suppressed mitochondrial-mediated apoptotic pathway. Moreover, NO, a typical pro-survival substance inhibiting apoptosis-related pathways, was upregulated by HMP 
A
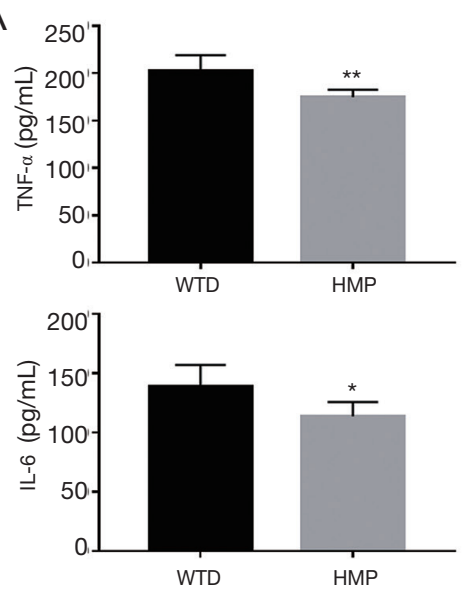

C
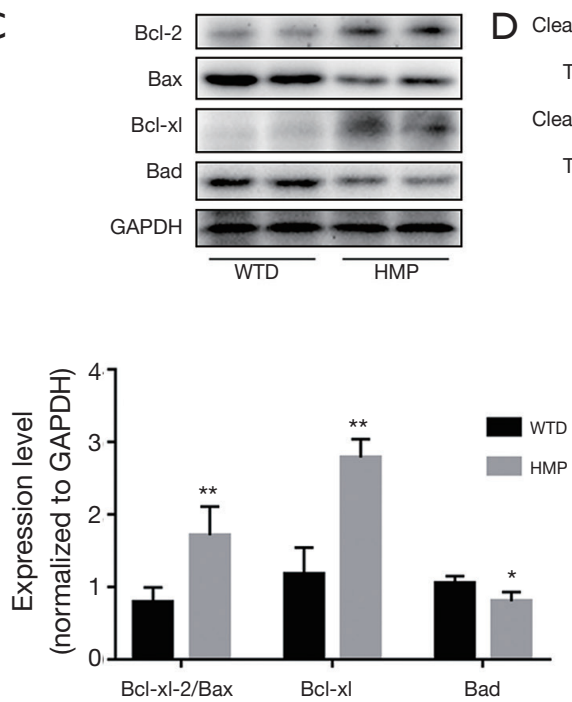
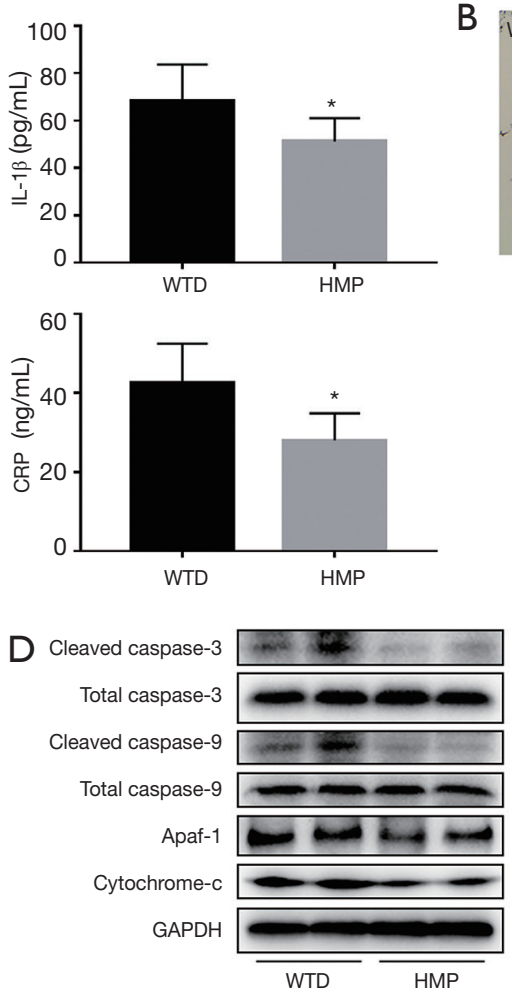

E

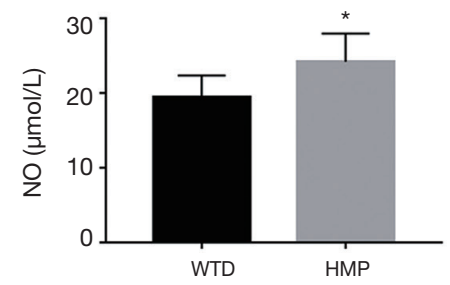

B
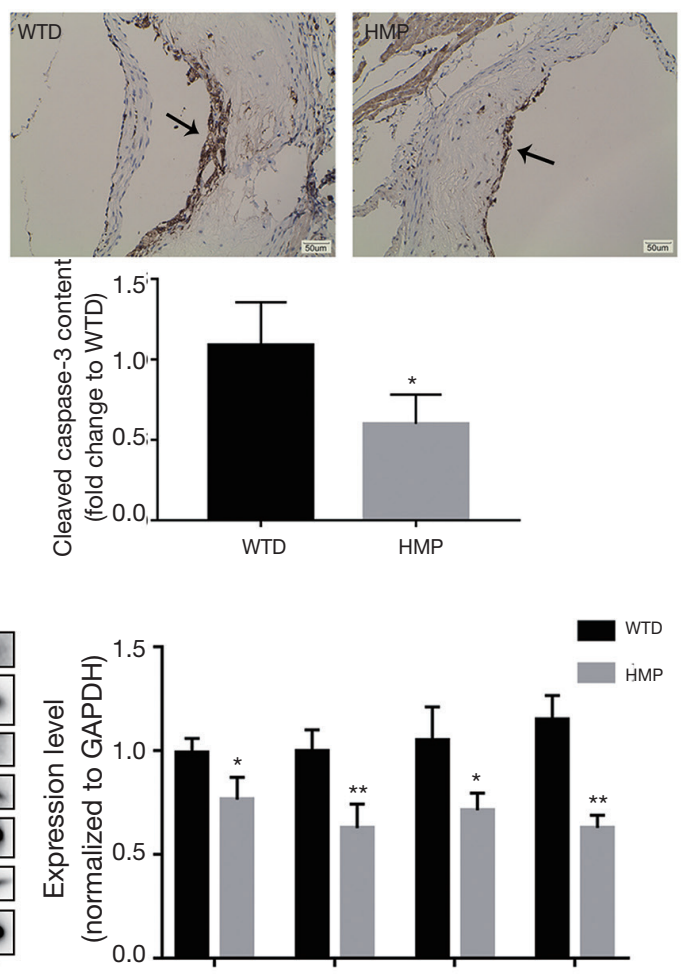

Figure 7 Oral gavage of heart-protecting musk pill (HMP) displayed protective effects against vascular wall injury. (A) Concentrations of serum TNF- $\alpha$, IL-1 $\beta$, IL-6 and CRP were detected by ELISA kits. (B) The expression of cleaved caspase-3. The arrows showed the positive area of cleaved caspase-3. (C) Protein levels of Bcl-2 family members in the aortic tissue. (D) The contents of signal proteins associated with mitochondrial-dependent apoptosis pathway. (E) The concentration of serum NO. Data were expressed as the mean \pm SD, $\mathrm{n}=6$ for $(\mathrm{A})$ and $(\mathrm{E})$, $\mathrm{n}=4$ for $(\mathrm{B}-\mathrm{D}) .{ }^{*} \mathrm{P}<0.05,{ }^{*} \mathrm{P}<0.01$ vs. WTD group. Scale bar $=50 \mu \mathrm{m}$.

treatment $(\mathrm{P}<0.05)$ (Figure $7 E)$.

\section{Discussion}

Pharmacological studies have demonstrated that several kinds of TCMs are effective in suppressing the development of atherosclerotic lesions (28). Consistent with previous studies, HMP, originating from the TCM Suhexiang pill, was found to decrease atheroma plaque burden in $\mathrm{apoE}^{-/-}$mice. For the first time, we discovered that atheroprotective mechanisms of HMP might be due to improvement of lipid metabolism and alleviation of vascular intimal injury (Figure 8).

In comparison with plaque enlargement-induced luminal stenosis, the plaque rupture-triggered arterial occlusion is more prone to cause life-threatening clinical events, such as acute myocardial infarction and cerebral stroke (29). Vulnerable plaques are characterized by large lipid-rich necrotic cores, decreased collagen amount, increased macrophage accumulation and smooth muscle cell reduction (30). According to these traits, HMP appeared to effectively enhance the stability of atheromatous lesions, as evidenced 


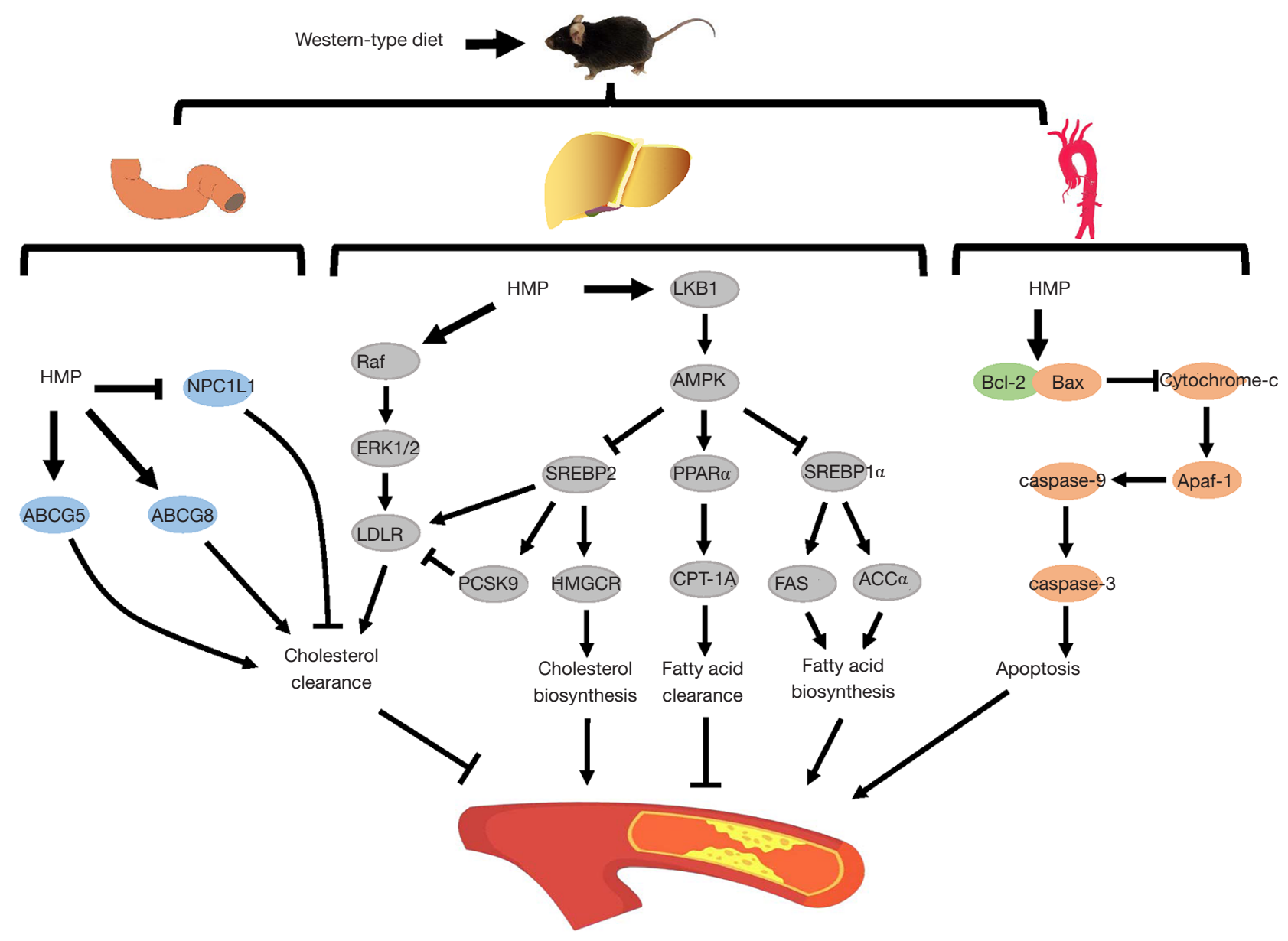

Figure 8 The schematic illustration of the potential molecular mechanisms by which heart-protecting musk pill (HMP) alleviated atherosclerosis development.

by shrink of necrotic core, escalation of collagen content, attenuation of macrophage infiltration and increase of smooth muscle cell, suggesting that HMP had therapeutic potentials for improving plaque destabilization.

Hypercholesterolemia has been demonstrated to be a pathological condition related to atherogenesis acceleration (2). Our results showed that HMP-treated mice presented TC and LDL-C level reduction in the bloodstream. Thereafter, we further explored the relevant mechanisms required for regulatory actions of HMP on cholesterol metabolism. HMGCR, regarded as a cholesterol synthesis rate-limiting enzyme, is targeted by several cholesterol-lowing drugs. LDLR acts as a membrane glycoprotein in hepatocytes and is responsible for removing circulating LDL-C. Moreover, PCSK9 is a serine protease inducing the lysosomal degradation of $\operatorname{LDLR}(23,24)$. It has been illustrated that these molecules are regulated by the same upstream signal protein SREBP2 (19). In this study, we identified that HMP potently lowered SREBP2 expression, followed by HMGCR and PCSK9 content reduction. It should be noted that the LDLR mRNA and protein levels were elevated in the HMP group compared to those in the WTD group. We speculated that this discrepant result might be attributed to HMP-induced activation of ERK1/2 pathway, given that ERK1/2 cascade could affect mRNA 3'-UTR structure which controlled stabilization of LDLR mRNA and several drugs including berberine and triciribine had been proved to increase the stability and content of $L D L R$ mRNA via stimulating ERK1/2 pathway through SREBP2independent processes $(20,31,32)$. Moreover, as PCSK9 regulated the elimination of LDLR protein (16), level reduction of PCSK9 induced by HMP treatment might lead to decrease in LDLR degradation in the lysosome compartment, thereby increasing the stability and content 
of LDLR protein. Thus, through modulating SREBP2regulated pathway and ERK1/2 cascade, HMP might inhibit cholesterol production and accelerate cholesterol uptake in hepatocytes, which resulted in TC and LDL-C concentration reduction in the bloodstream, consequently improving risks of atherosclerosis progression.

It has been proved that atheroprone effects of TG overload are largely due to the induction of inflammation, aggregation of endothelial dysfunction and impairment of fibrinolysis (33). Our results showed that HMP had favorable impacts on lowering blood TG level and improving lipid accumulation in liver tissues. Next, relevant mechanisms necessary for regulatory effects of HMP on TG content were assessed. Fatty acid $\beta$-oxidation is a biological process that enables TG consumption and nuclear receptor PPAR $\alpha$ has pivotal roles in this process. Once stimulated, PPAR $\alpha$ promotes the expression of target gene CPT-1A, which catalyzed long-chain acyl-COA migration from the cytosol to the mitochondrial matrix for oxygenolysis (21). We observed that HMP induced the level increase of PPAR $\alpha$, followed by up-regulation of CPT-1A. It is verified that de novo lipogenesis is an efficient way favoring TG level increase. SREBP1c is a transcriptional factor that possesses crucial roles in de novo lipogenesis via mediating expressions of downstream lipogenic gene $F A S$ and $A C C \alpha$. There is evidence that high expression of SREBP1c causes circulating TG accumulation and hepatic steatosis $(34,35)$. Our results showed that HMP notably reduced the level of SREBP1c and the expression of FAS and ACC $\alpha$. Hence, mediation of signal pathways involved in lipid $\beta$-oxidation and de novo lipogenesis might contribute to the regulatory roles of HMP in TG concentration of WTD-fed apoE ${ }^{-/-}$mice.

AMPK is an intracellular energy sensor that exerts key effects on lipid and glucose metabolism (23). When activated, AMPK switches off anabolic activities including cholesterol and fatty acid biosynthesis, and switches on catabolic processes like fatty acid oxidation through regulating relevant signaling factors, such as up-regulation of PPAR $\alpha$ and decrease of SREBP2 and SREBP1c level (36). Similar to results reported by other studies, the enhanced activity of AMPK was seen in the hepatocytes of HMPtreated mice with improved lipid profiles. Moreover, HMGCR and ACC $\alpha$ are well identified downstream targets of AMPK and a pivotal mechanism underlying AMPKinduced lipid production inhibition is due to the induction of phosphorylation of HMGCR and ACC $\alpha$, the inactive form of the two enzymes (23). Our findings showed that, consistent with AMPK modification, the phosphorylated HMGCR and ACC $\alpha$ levels were markedly increased in the HMP group. Regarding the upstream kinases responsible for AMPK activation, LKB1 and CaMKK $\beta$ are two major upstream regulators controlling Thr-172 phosphorylation on the $\alpha$-subunit of AMPK. It has been verified that LKB1 and CaMKK $\beta$ mainly activate AMPK in response to energy stress increased intracellular calcium, respectively $(36,37)$. In this study, p-LKB1 rather than $\mathrm{p}-\mathrm{CaMKK} \beta$ showed the same tendency of level changes with p-AMPK in the hepatocytes of HMP-treated mice, indicating that HMP might affect LKB1-mediated AMPK activation to regulate pathways implicated in lipid metabolism, eventually leading to improvement of blood lipid profiles.

Except for the liver, the intestine is regarded as an important organ responsible for lipid metabolism. By modulating dietary lipid absorption and internal lipid excretion, the intestine indirectly impacts the regulation of circulating lipid profiles (24). We found that HMP raised the fecal TC content, without changing TG level. It is reported that cholesterol absorption from the intestinal lumen is controlled by NPC1L1 in the enterocytes. Several atherogenic factors have been found to increase the expression of intestinal NPC1L1 and then elevate the levels of blood cholesterols $(24,38)$. In addition, level reduction of NPC1L1 is reported to be an effective way to inhibit cholesterol absorption, thereby ameliorating dyslipidemia and suppressing atherosclerosis progression $(39,40)$. There is evidence that cholesterol secretion from enterocytes to the gut lumen is regulated by apical transporters ABCG5 and ABCG8 (16,24). Findings from previous researches demonstrate that up-regulation of ABCG5 and ABCG8 is able to exert atheroprotective roles via enhancing cholesterol excretion and then decreasing the level of blood cholesterol $(38,41)$. As shown in Figure 6, HMP was likely to increase fecal TC level by promoting transintestinal cholesterol excretion and weakening its influx, which might partly mitigate the accumulation of cholesterol in the circulation and then improve the development of atherosclerosis.

Upon pathological stimulation, vascular endothelial cells (ECs) produce an apoptotic phenotype, which causes intima injury, followed by progression of atheromatous lesions $(7,27)$. Our results revealed that HMP gavage alleviated ECs apoptosis in the area of aortic wall. As inflammation could induce atherogenesis by causing vascular intimal damage $(25,42)$, we analyzed the effects of HMP on inflammation development and found that HMP reduced 
levels of TNF- $\alpha$, IL-1 $\beta$, IL- 6 , and CRP in the bloodstream. Furthermore, NO, as a bioactive factor responsible for cellular survival (43), was found to be upregulated in the circulation of HMP-treated mice. These data elucidated the vasoprotective properties of HMP. The Bcl-2 family contains regulatory proteins that either trigger or suppress apoptosis. Among them, Bax oligomerizes and forms pores that permeabilize the mitochondrial outer membrane and cause leakage of apoptogenic factors. In contrast, Bcl-2 forms heterodimers with Bax to prevent Bax oligomerization. Moreover, Bcl-xl is capable of repressing Bax activation, whereas the function of Bad is to inhibit activities of antiapoptotic proteins (44). We discovered that HMP treatment induced increment of $\mathrm{Bcl}-2 / \mathrm{Bax}$ ratio and $\mathrm{Bcl}-\mathrm{xl}$ content and triggered decrement of Bad level. Once released in the cytosol, the apoptogenic molecules, particularly cytochrome-c, sequentially activate Apaf- 1 and downstream caspase-9, followed by caspase- 3 cleavage and apoptosis initiation (44). In our study, there was a decrease in levels of cytoplasm cytochrome-c, Apaf-1 and cleaved caspase-9, 3 in the arterial walls of the HMP group. These data verified that HMP possibly displayed anti-atherogenic actions via improving vascular intimal injury by abating mitochondrialrelated apoptosis signaling cascade. However, it is not clear whether HMP directly improves vascular damage or not. Moreover, the specific mechanisms by which HMP inhibits the activation of apoptotic pathway in the arterial wall are poorly understood. These deficiencies are needed to be settled using the in vitro model in the near future.

In summary, our study demonstrates that HMP intervention considerably ameliorates the development of atheromatous leisons in apoE $\mathrm{E}^{-/-}$mice fed by WTD and the atheroprotective effects are due to the regulation of lipid metabolism as well as inhibition of vascular intimal damage. These data provide novel insights into the molecular mechanisms underlying HMP attenuates atherosclerosisrelated diseases.

\section{Acknowledgments}

Funding: This study was supported by the grants from the National Natural Science Foundation of China (No. 81873518 and No. 81270353).

\section{Footnote}

Conflicts of Interest: The authors have no conflicts of interest declare.
Ethical Statement: The authors are accountable for all aspects of the work in ensuring that questions related to the accuracy or integrity of any part of the work are appropriately investigated and resolved. All animal procedures were approved by the Institutional Animal Care and Use Committee of Tongji Medical College, Huazhong University Science and Technology, Wuhan, China. All experiment procedures were conformed to the National Institutes of Health Guide for the Care and Use of Laboratory Animals.

\section{References}

1. Herrington W, Lacey B, Sherliker P, et al. Epidemiology of atherosclerosis and the potential to reduce the global burden of atherothrombotic disease. Circ Res 2016;118:535-46.

2. Sathiyakumar V, Kapoor K, Jones SR, et al. Novel therapeutic targets for managing dyslipidemia. Trends Pharmacol Sci 2018;39:733-47.

3. Kosmas CE, Silverio D, Sourlas A, et al. Antiinflammatory therapy for cardiovascular disease. Ann Transl Med 2019;7:147.

4. Niu N, Xu S, Xu Y, et al. Targeting mechanosensitive transcription factors in atherosclerosis. Trends Pharmacol Sci 2019;40:253-66.

5. Catapano AL, Graham I, De Backer G, et al. 2016 ESC/ EAS guidelines for the management of dyslipidaemias. Eur Heart J 2016;37:2999-3058.

6. Luo Y, Lu S, Ai Q, et al. SIRT1/AMPK and Akt/eNOS signaling pathways are involved in endothelial protection of total aralosides of Aralia elata (Miq) Seem against high-fat diet-induced atherosclerosis in ApoE-/- mice. Phytother Res 2019;33:768-78.

7. Haybar H, Shahrabi S, Rezaeeyan H, et al. Endothelial cells: from dysfunction mechanism to pharmacological effect in cardiovascular disease. Cardiovasc Toxicol 2019;19:13-22.

8. Stroes ES, Thompson PD, Corsini A, et al. Statinassociated muscle symptoms: impact on statin therapyEuropean Atherosclerosis Society Consensus Panel Statement on Assessment, Aetiology and Management. Eur Heart J 2015;36:1012-22.

9. Nissen SE, Stroes E, Dent-Acosta RE, et al. Efficacy and tolerability of evolocumab vs ezetimibe in patients with muscle-related statin intolerance. JAMA 2016;315:1580.

10. Lu L, Sun X, Chen C, et al. Shexiang Baoxin Pill, derived from the traditional Chinese medicine, provides protective roles against cardiovascular diseases. Front Pharmacol 
2018;9:1161

11. Zhao JH, Zhang L, Liu Y, et al. Effect of Shexiang Baoxin Pill in alleviating early hypertensive renal injury in rats. Chin J Integr Med 2019. [Epub ahead of print].

12. Yu J, Gao C, Ren Y. Effect of Shexiang Baoxin pill on abdominal aortic atherosclerosis in rabbits. J Chin Pract Diagn Treat 2012;26:852-6.

13. Huang F, Liu Y, Yang X, et al. Shexiang Baoxin pills promotes angiogenesis in myocardial infarction rats via upregulation of 20-HETE-mediated endothelial progenitor cells mobilization. Atherosclerosis 2017;263:184-91.

14. Xu M, Zhang $Z$. The effects and mechanisms of ShexiangBaoxinwan on myocardial fibrosis in rats with pressure overload. Pharmacology and Clinics of Chinese Materia Medica 2016;32:128-32.

15. Tao Y, Li J, Wei Y, et al. Experimental study of Shexiang Baoxin pill in preventing and treating atherosclerosis. Tradit Chin Drug Res Clin Pharmacol 2015;26:508-11.

16. Wei D, Zheng N, Zheng L, et al. Shexiang Baoxin Pill corrects metabolic disorders in a rat model of metabolic syndrome by targeting mitochondria. Front Pharmacol 2018;9:137.

17. Xiang L, Jiang P, Zhan C, et al. The serum metabolomic study of intervention effects of the traditional Chinese medicine Shexiang Baoxin Pill and a multi-component medicine polypill in the treatment of myocardial infarction in rats. Mol Biosyst 2012;8:2434-42.

18. Gluchowski NL, Becuwe M, Walther TC, et al. Lipid droplets and liver disease: from basic biology to clinical implications. Nat Rev Gastroenterol Hepatol 2017;14:343-55.

19. Vantaggiato C, Panzeri E, Citterio A, et al. Antipsychotics promote metabolic disorders disrupting cellular lipid metabolism and trafficking. Trends Endocrinol Metab 2019;30:189-210.

20. Bjune K, Wierod L, Naderi S. Triciribine increases LDLR expression and LDL uptake through stabilization of LDLR mRNA. Sci Rep 2018;8:16174.

21. Yu XH, Zheng X, Tang C. Peroxisome proliferatoractivated receptor $\alpha$ in lipid metabolism and atherosclerosis. Adv Clin Chem 2015;71:171-203.

22. Buñay J, Baron S, Lobaccaro JA. LXRs are finally being adequately targeted in atherosclerosis. Ann Transl Med 2018;6:S28.

23. Day EA, Ford RJ, Steinberg GR. AMPK as a therapeutic target for treating metabolic diseases. Trends Endocrinol Metab 2017;28:545-60.

24. Yu XH, Zhang D, Zheng X, et al. Cholesterol transport system: an integrated cholesterol transport model involved in atherosclerosis. Prog Lipid Res 2019;73:65-91.

25. Raggi P, Genest J, Giles JT, et al. Role of inflammation in the pathogenesis of atherosclerosis and therapeutic interventions. Atherosclerosis 2018;276:98-108.

26. Shen Y, Song J, Wang Y, et al. M2 macrophages promote pulmonary endothelial cells regeneration in sepsis-induced acute lung injury. Ann Transl Med 2019;7:142.

27. Gimbrone MA Jr, Garcia-Cardena G. Endothelial cell dysfunction and the pathobiology of atherosclerosis. Circ Res 2016;118:620-36.

28. Wang C, Niimi M, Watanabe T, et al. Treatment of atherosclerosis by traditional Chinese medicine: questions and quandaries. Atherosclerosis 2018;277:136-44.

29. Perrotta P, Emini Veseli B, Van der Veken B, et al. Pharmacological strategies to inhibit intra-plaque angiogenesis in atherosclerosis. Vascul Pharmacol 2019;112:72-8.

30. Finn AV, Nakano $M$, Narula J, et al. Concept of vulnerable/unstable plaque. Arterioscler Thromb Vasc Biol 2010;30:1282-92.

31. Kong W, Wei J, Abidi P, et al. Berberine is a novel cholesterol-lowering drug working through a unique mechanism distinct from statins. Nat Med 2004;10:1344-51.

32. Yashiro T, Nanmoku $M$, Shimizu $M$, et al. 5-Aminoimidazole-4-carboxamide ribonucleoside stabilizes low density lipoprotein receptor mRNA in hepatocytes via ERK-dependent $\mathrm{HuR}$ binding to an AU-rich element. Atherosclerosis 2013;226:95-101.

33. Reiner $\check{Z}$. Hypertriglyceridaemia and risk of coronary artery disease. Nat Rev Cardiol 2017;14:401-11.

34. Xu J, Peng Y, Zeng Y, et al. 2, 3, 4', 5-tetrahydroxystilbene2-0-beta-d glycoside attenuates age- and diet-associated non-alcoholic steatohepatitis and atherosclerosis in LDL receptor knockout mice and its possible mechanisms. Int J Mol Sci 2019. doi: 10.3390/ijms20071617.

35. Ren L, Sun D, Zhou X, et al. Chronic treatment with the modified Longdan Xiegan Tang attenuates olanzapineinduced fatty liver in rats by regulating hepatic de novo lipogenesis and fatty acid $\beta$-oxidation-associated gene expression mediated by SREBP-1c, PPAR- $\alpha$ and AMPK- $\alpha$. J Ethnopharmacol 2019;232:176-87.

36. Herzig S, Shaw RJ. AMPK: guardian of metabolism and mitochondrial homeostasis. Nat Rev Mol Cell Biol 2018;19:121-35.

37. Li YC, Qiao JY, Wang BY, et al. Paeoniflorin ameliorates fructose-induced insulin resistance and hepatic steatosis 
by activating LKB1/AMPK and AKT pathways. Nutrients 2018;10. doi: 10.3390/nu10081024.

38. Wang HH, Garruti G, Liu M, et al. Cholesterol and lipoprotein metabolism and atherosclerosis: recent advances in reverse cholesterol transport. Ann Hepatol 2017;16:S27-42.

39. Ontawong A, Duangjai A, Muanprasat C, et al. Lipidlowering effects of Coffea arabica pulp aqueous extract in Caco-2 cells and hypercholesterolemic rats. Phytomedicine 2019;52:187-97.

40. Yin J, Wang J, Li F, et al. The fucoidan from the brown seaweed Ascophyllum nodosum ameliorates atherosclerosis in apolipoprotein E-deficient mice. Food Funct

Cite this article as: $\mathrm{Lu} \mathrm{L}$, Qin Y, Chen C, Zhang X, Xu X, Lv C, Wan X, Ruan W, Guo X. The atheroprotective roles of heartprotecting musk pills against atherosclerosis development in apolipoprotein E-deficient mice. Ann Transl Med 2019;7(23):714. doi: 10.21037/atm.2019.12.22
2019;10:5124-39.

41. Patel SB, Graf GA, Temel RE. ABCG5 and ABCG8: more than a defense aganist xenosterols. J Lipid Res 2018;59:1103-13.

42. Sima P, Vannucci L, Vetvicka V. Atherosclerosis as autoimmune disease. Ann Transl Med 2018;6:116.

43. Chen JY, Ye Z, Wang X, et al. Nitric oxide bioavailability dysfunction involves in atherosclerosis. Biomed Pharmacother 2018;97:423-8.

44. Czabotar PE, Lessene G, Strasser A, et al. Control of apoptosis by the BCL-2 protein family: implications for physiology and therapy. Nat Rev Mol Cell Biol 2014;15:49-63. 